\title{
A Naturalistic Driving Study of Feedback Timing and Financial Incentives in Promoting Speed Limit Compliance
}

\author{
Huei-Yen Winnie Chen and Birsen Donmez
}

Version Accepted Manuscript

Citation H. -Y. W. Chen and B. Donmez, "A Naturalistic Driving Study of

(published version) Feedback Timing and Financial Incentives in Promoting Speed Limit Compliance," in IEEE Transactions on Human-Machine Systems, vol. 52, no. 1, pp. 64-73, Feb. 2022, doi: 10.1109/THMS.2021.3117234.

Publisher's Statement (C) 2021 IEEE. Personal use of this material is permitted. Permission from IEEE must be obtained for all other uses, in any current or future media, including reprinting/republishing this material for advertising or promotional purposes, creating new collective works, for resale or redistribution to servers or lists, or reuse of any copyrighted component of this work in other works.

How to cite TSpace items

\begin{abstract}
Always cite the published version, so the author(s) will receive recognition through services that track citation counts, e.g. Scopus. If you need to cite the page number of the author manuscript from TSpace because you cannot access the published version, then cite the TSpace version in addition to the published version using the permanent URI (handle) found on the record page.
\end{abstract}

This article was made openly accessible by $U$ of $T$ Faculty. Please tell us how this access benefits you. Your story matters. 


\title{
A Naturalistic Driving Study of Feedback Timing and Financial Incentives in Promoting Speed Limit Compliance
}

\author{
Huei-Yen Winnie Chen and Birsen Donmez, Senior Member, IEEE
}

\begin{abstract}
Inappropriate speed choice increases crash risk. Emerging technologies, such as in-vehicle systems, can provide real-time and post-drive feedback to alert or educate drivers of their unsafe speed choices. Financial incentives, such as insurance reductions, can also be integrated with feedback. Although previous research supports the benefits of these approaches, research is limited in establishing their relative effectiveness and their long-term impact on speed limit compliance. A naturalistic driving study with 58 participants was conducted to investigate the effects of post-drive feedback versus financial incentives, both provided in conjunction with real-time feedback, on mitigating speeding behaviours. The study included a 4-week long baseline data collection period (no feedback), a 10-week intervention period examining three feedback types in a between subjects design (real-time only; real-time and financial incentives; and realtime and post-drive feedback), and a 2 -week post-intervention period (feedback removed). Both real-time feedback alone and in conjunction with financial incentives were effective in raising speed limit compliance, but the effects did not sustain after the removal of feedback/incentives. Post-drive feedback, which was provided in the vehicle after a trip and as aggregated feedback on a website, did not appear to provide any benefits towards speed limit compliance; potential factors, including (voluntary) access frequency of post-drive feedback website, are discussed. Future research should continue to explore the feedback/incentive design space-carefully considering individual differences in driver characteristics and motivations-for supporting speed limit compliance and other risky driving behaviours.
\end{abstract}

Index Terms-Driver safety, financial incentives, naturalistic driving study, post-drive feedback, speeding.

\section{INTRODUCTION}

I NAPPROPRIATE speed choice is a well-established factor of increased crash risk [1], [2]. In 2015, NHTSA reported the highest year to year increase in motor vehicle crash fatalities in the United States since the 60s, and this number continued to raise, with 37,461 fatalities reported in 2016 [3]. Nearly a third of these fatalities resulted from speeding-related crashes, a $4 \%$ increase from 2015.

Manuscript received: October 25, 2018; Revised: February 28, 2020 and October 6, 2020; Accepted September 19, 2021.

This paper was recommended for publication by Editor David Kaber upon evaluation of the Associate Editor and Reviewers' comments.

The funding for this work was provided by the Toyota Collaborative Safety Research Center (CSRC) and Auto 21 Network of Centres of Excellence.

H.-Y. W. Chen was with the University of Toronto, Toronto, Canada. She is now with the Department of Industrial \& Systems Engineering, State University of New York at Buffalo (e-mail: winchen@buffalo.edu).

B. Donmez, is with the Department of Mechanical and Industrial Engineering, University of Toronto, Toronto, ON, Canada (e-mail: donmez@mie.utoronto.ca).
Given the high prevalence of speeding related fatalities, countermeasures for speeding are vast and varied, including law enforcement, driver education, and engineering solutions through vehicle or traffic infrastructure design (see [4], [5] for examples and effectiveness of various measures). In general, speeding countermeasures are designed to provide feedback regarding the driver's speed choices. Owing to the rapid advancement in sensor technology and increases in computational power, in-vehicle driver support systems are increasingly popular for providing real-time feedback to reduce speeding. These Intelligent Adaptive Systems (IAS) aim to help drivers adapt their speed to a static or dynamic speed limit via some combination of visual, auditory, and/or haptic feedback in real time. Field studies have found these systems to be effective in reducing speeding behaviours, e.g., [6]-[8], however, their effectiveness may not persist over time, especially when the systems cease to be available [9].

A European field study [6] compared two kinds of IAS systems: support via an active accelerator pedal, and a warning system using beep signals and a flashing red light when the speed limit was exceeded. While both systems reduced the mean and $85^{\text {th }}$ percentile speeds, with the active accelerator pedal being more effective of the two, there was no long-lasting effect when the systems were removed. In some cases, removal of real-time feedback may lead to a boomerang effect, where drivers speed up to compensate for the reduced speed earlier. Boyle and Mannering [10] showed that traffic advisory information regarding adverse weather conditions may reduce speeds temporarily, but drivers tend to compensate for lost time by increasing speeds downstream when the real-time information is no longer available or valid. Technology acceptance is also an issue, as drivers may override such systems [11]. In [6], seven out of 20 participants indicated that they would not keep the IAS system even though the technology acceptance rating results were generally positive towards the system.

Given these potential limitations of real-time feedback, researchers have investigated other means to motivate speeding reductions, such as the use of financial incentives as a positive reinforcement for speed limit compliance. Mullen, Maxwell and Bedard [12] conducted a driving simulator study to investigate the amount of speed reduction that could be achieved when real-time, visual feedback was paired with a point-reward system that awarded up to $\$ 10$ in gift cards for speed limit compliance. Reagan, Bliss, Houten and Hilton [13] instrumented eight vehicles and recruited 50 participants to examine the effectiveness of real-time feedback and monetary incentives over a four week period. In both studies, real-time feedback alone showed no significant improvement over the 
control condition, but financial incentives alone and financial incentives paired with real-time feedback were similarly effective in achieving significant speed reduction.

Post-drive feedback, which refers to delayed feedback provided to the driver after a trip, has also been investigated along with real-time feedback and monetary incentives. A simulator study examined the effectiveness of a Pay-As-YouDrive (PAYD) insurance scheme, which associates an individual driver's behaviour to their insurance fees [14]. Feedback on both the driving behaviour (speeding, accelerating, braking, and steering) and the consequential changes in insurance fees were delivered in real-time, or postdrive on a website, to two respective groups of participants. While both types of feedback led to improved driving behaviour, the study did not assess long term impact of feedback, especially when feedback is removed. Similar studies have also been carried out in the real world. A field study conducted in Winnipeg, Manitoba provided monetary incentives along with real-time and post-drive feedback on speeding and tailgating behaviours [15]. Weekly reports of their related driving information (e.g., compliance rates and fuel economy), as well as their accumulated reward points were provided to the participants on a secured website. The results demonstrated potential benefits of a feedback-reward system especially promising for those who were less compliant to begin with. Another field study conducted in the Netherlands also found that monetary incentives and post-drive feedback for speed limit compliance in the form of insurance reduction was effective in reducing young drivers' speeds during the intervention, but not after [16]. While these studies have all found post-drive feedback along with monetary rewards to be effective in curbing unsafe driving behaviour, there was no control in the study design to inform on the relative effectiveness of financial incentives compared to post-drive feedback on driving behaviour.

Only one study that we could identify studied the effectiveness of post-drive feedback alone for speeding behaviours. In [17], a field study was conducted to validate a diagnostic system, which provided various driver safety metrics, including the extent and duration of exceeding predefined speed limits as well as exposure statistics (e.g., distance and time travelled) to participants (employees of two companies driving company-owned cars) through a personalized website. In addition, the website also showed metrics aggregated over all other study participants, and each participant received a personal information session to discuss their driving behaviour before the feedback system was activated. While components of this feedback system (inperson training vs. post-drive feedback, or individual vs. group metrics) were not assessed independently, the study showed that exposure to feedback had positive impact on the participants. However, the positive impact did not sustain over time: the web-based feedback system initially received high levels of attention from the participants, but the number of logins decreased as time progressed.

The studies cited above have generally supported, to various extents, the usefulness of driver feedback and incentives on speed limit compliance. However, to the best of our knowledge, there is no study that differentiated the effects of financial incentives from post-drive feedback. Evidence about the long term impacts of these interventions have also been limited in previous work, as many of these studies had fairly short timeframes (e.g., [14] was a simulator study that participants spent only a couple hours in total, and in [13], the intervention lasted only two weeks, followed by one more week of data collection post-intervention).

The study reported herein addressed the gaps in previous literature by collecting naturalistic driving data over a relatively long timeframe (16 weeks total) to assess and compare the effectiveness of adding financial incentives to real-time feedback and adding post-drive information to real-time feedback in improving speed limit compliance, and whether any benefits observed would sustain over time, during and after the removal of feedback and/or incentives.

\section{METHOD}

Compared to the more traditional methods in driver behavioural research (e.g., simulator studies, instrumented car studies, and self-reported data analysis), naturalistic driving studies (NDS) - the unobtrusive data collection about the driver, the vehicle, and the surroundings during everyday trips-have the distinct advantage of being able to provide insights into driver behaviours in their naturalistic environment without the presence of experimenters or rigorous controls [18]. At the same time, the lack of experimental control is difficult for a pure NDS to draw conclusions regarding causal relationships between driver behaviours and outcomes.

In this study, we collected naturalistic driving data in a quasiexperiment to assess the effectiveness of various feedback/incentive designs for encouraging speed limit compliance. Apart from assigning participants into an experimental group, which determined the combination of feedback and/or incentives they would receive, no further experimental controls were exerted. Participants, while recruited from a particular region, were not restricted to any amount of driving or the location of their travels during the study period, nor were the use (viewing/exploring) of feedback enforced. Such experimental controls would potentially yield stronger causal inferences, but would have prevented us from observing naturalistic behaviours in the presence/absence of our feedback systems.

\section{A. Study Site and Population}

Naturalistic data collection took place in 2015, in the Winnipeg metropolitan area in Manitoba, Canada. The Winnipeg metropolitan area includes the City of Winnipeg and eleven rural municipalities for a total of $5,306.79 \mathrm{~km}^{2}$ in land area, and was estimated to have a population of 778,489 (population density of 146.7 persons per $\mathrm{km}^{2}$ ) in 2016 [19]. If participants drove beyond this region, speed limit information was not available, and the feedback systems tested were not functional. Although we did not limit where participants may travel during the study, the bulk of travel observed occurred within the City of Winnipeg (see Results), thus the analysis reported in this paper focused on data collected within this urban environment.

\section{B. Participants}

In total, 60 participants were recruited for the study, but one 
withdrew from the study prior to data collection, and another withdrew after four weeks of data collection. Fig. 1 presents the experimental design, along with participant demographics for the 58 participants who completed the study.

Participants were recruited using online advertisements and email messages forwarded to local communities in Winnipeg. To be eligible for the study, participants had to have a valid full driver's license from Manitoba, and be between the ages of 25 and 40. This particular age range was chosen to control for agerelated variability in speed limit compliance [20], [21], and exclude novice and older drivers from this initial study. Additional criteria recruited participants who drove $200 \mathrm{~km}$ or more per week or fill their gas tank once a week; drove in both the City of Winnipeg and the surrounding regions; had access to email and the internet; and were the sole or primary driver of an insured vehicle. To ensure that vehicles would be compatible with the instrumentation system (see Apparatus), participating vehicles had to be gas-fueled, with a model year between 2004 and 2014 (inclusively); and they had to have no automated driving aids, such as adaptive cruise control or lane departure systems. Furthermore, only one participant per family could take part in the study and candidates who had previously participated in any naturalistic driving study were excluded.

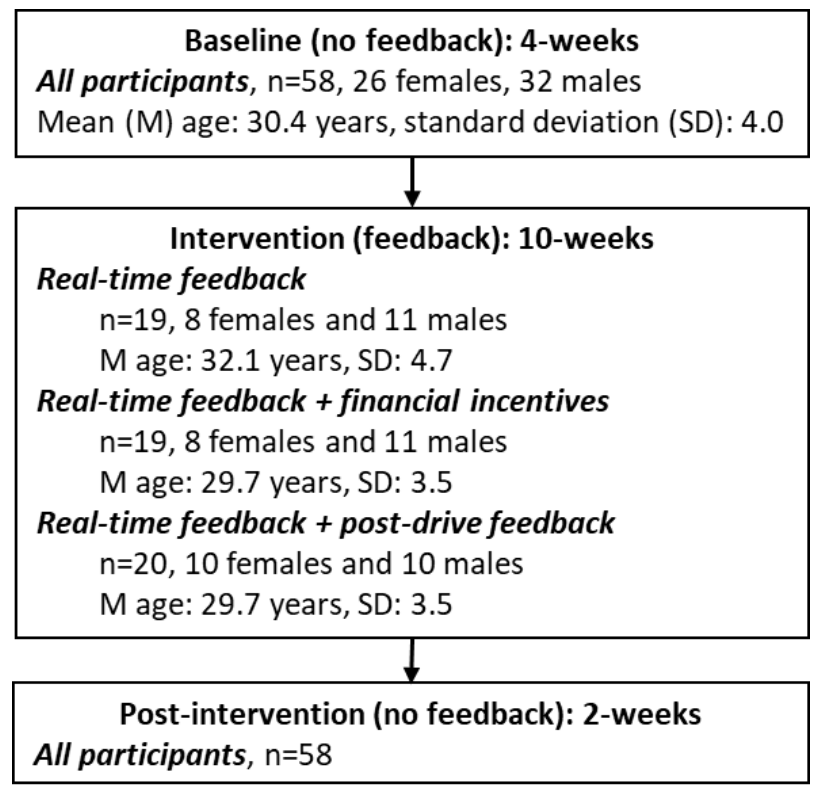

Fig. 1. Experimental design and participant demographics.

\section{Experimental Design}

Participants were randomly assigned, ensuring a rough balance on gender, to one of the three speed limit compliance feedback conditions: real-time feedback only, real-time feedback and financial incentives, real-time feedback and postdrive feedback. A control group with no feedback or incentives was not included as previous literature has well established the effectiveness of real-time feedback (e.g., [6]-[8]). Instead, our study assessed the potential benefits of adding financial incentives vs. post-drive feedback, to real-time feedback.

As shown in Fig. 1, all participants underwent a baseline period, followed by an intervention period, and a postintervention period. Data collection (16 weeks total) took place from early June to mid-September in 2015, where weather conditions were relatively consistent to avoid confounding due to seasonal effects (e.g., heavy snowfall, spring flooding).

Participants received $C \$ 500$ upon completion of the study regardless of the feedback condition they were assigned to and regardless of how they performed in the study. However, participants of different feedback conditions received different information about the compensation scheme prior to the study. Real-time only feedback participants were offered C $\$ 500$ compensation for completing the study. Participants assigned to the financial incentives condition were informed that they would receive $\mathrm{C} \$ 200$ for completing the study, and an additional bonus of up to $\mathrm{C} \$ 300$ based on their speed limit compliance during the intervention period. Participants of the post-drive feedback condition were told that they would receive $\mathrm{C} \$ 400$ upon completion of the study, and an additional bonus of up to $C \$ 100$ for accessing the feedback website regularly during the intervention period. Participants were debriefed at the end of the study regarding the actual compensation scheme. Ethics approval was obtained from the University of Toronto's Research Ethics Board.

\section{Apparatus}

Participants' vehicles were instrumented with PersenTech OttoView CVS44, a system that accessed vehicular data through an on-board diagnostics interface (OBDII), assessed speed limit compliance using GPS technology and pre-loaded posted speed limit information, and provided real-time visual feedback and post-drive trip summary via a LCD display that has a $70 \mathrm{~mm} \times 52.5 \mathrm{~mm}$ active area and a resolution of $320 \mathrm{x}$ 240 pixels (Fig. 2). The device stored data locally, and periodically transmitted data to a remote webserver using a GSM (a wireless data system)-GPRS (General Packet Radio System) radio and a TCP/IP connection. Vehicular data and trip information, including date, time, GPS location, speed, speed limit, and trip duration and distance, were recorded at $1 \mathrm{~Hz}$. Transmission of real-time data from the device to the webserver-database was configured to take place every 5 minutes during a trip, and a summary of the trip data (e.g., total distance travelled) was transmitted to the webserver-database at the end of the trip. Transmission of data during the study period allowed us to monitor data collection closely and enabled automatic generation of aggregated information for in-vehicle and web-based post-drive feedback. However, data analysis reported below were conducted using the full set of data collected at $1 \mathrm{~Hz}$.

\section{E. Feedback Designs}

The in-vehicle technology experienced by the participants (whether real-time or real-time + post-drive trip information) was referred to as the "SafeMiles System" and the post-drive feedback website was referred to as the "SafeMiles Website" for the purposes of communicating with the participants during the study and collecting their perceptions of and attitudes towards the different feedback designs that were tested. The feedback designs were created by the authors and research staff and underwent several rounds of revisions based on heuristic evaluations by several industry and academic experts of driver safety and/or usability. Furthermore, to ensure that real-time feedback did not impose significant visual demands on the 
driver, a pilot test was carried out to confirm that feedback is easy to understand and can be interpreted quickly: test participants were able to assess the situation with a presentation time as low as $250 \mathrm{~ms}$.

\section{1) Real-time feedback}

During the intervention period, all participants received visual real-time feedback of their speed limit compliance via the in-vehicle display. The display showed two elements: current speed of the vehicle and the posted speed limit at the current location (Fig. 2, top), both displayed in $\mathrm{km} / \mathrm{h}$, standard unit in Winnipeg. A change of background color (from white to orange) was designed to alert the driver when they exceed the posted speed limit by $5 \mathrm{~km} / \mathrm{h}$ or more (Fig. 2, top); the $5 \mathrm{~km} / \mathrm{h}$ tolerance was included based on industry experts' advice that speedometers in the vehicles are often inaccurate.

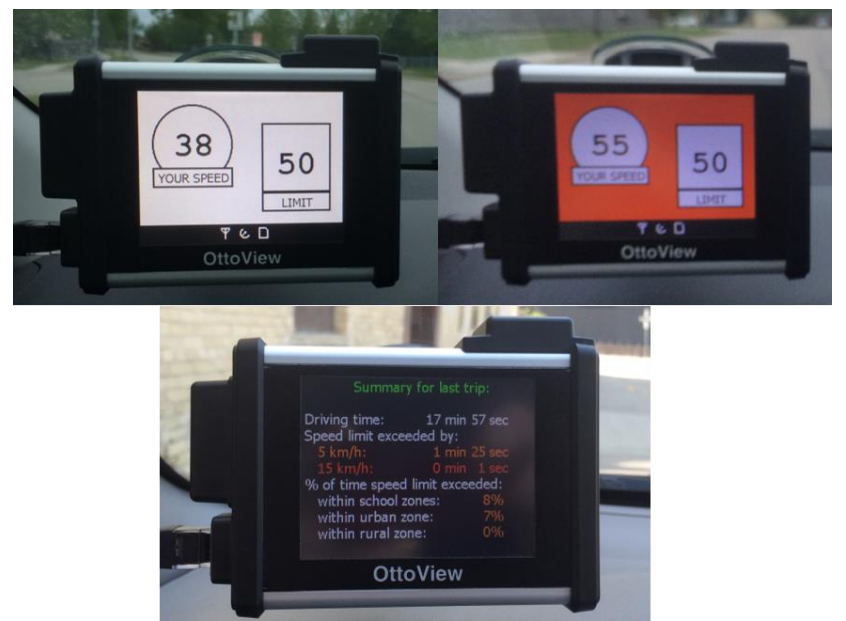

Fig. 2. (Top left) Real-time driver feedback indicating current speed and posted speed limit compliant with posted speed limit, and (top right) not compliant with posted speed limit. (Bottom) Post-drive trip summary.

\section{2) Post-drive feedback}

Post-drive feedback regarding speed limit compliance came in two forms: a single trip summary available on the in-vehicle display immediately after a trip, and a website with personal access for aggregated information over the intervention period. The trip summary was designed to inform drivers about their speed limit compliance in the preceding trip. In addition to total trip time and total time spent speeding (over the posted speed limit by $5 \mathrm{~km} / \mathrm{h}$ or more), the post-drive summary also indicated total time spent over the speed limit by $15 \mathrm{~km} / \mathrm{h}$ or more. These calculations excluded time spent idling, parking, or when the driver was unable to move due to heavy traffic. The post-drive summary also differentiated speeding within different environments (urban: City of Winnipeg, rural: surrounding regions of Winnipeg, and school zones, see Fig. 2 bottom).

The post-drive feedback website (see Fig. 3) presented individual participant's speed limit compliance data aggregated over time, in various timescales: overall change since the intervention period began; weekly compliance rates; and compliance rates over the last seven days. Various visualizations were used to present the information: text-based, bar graphs, and line trends. The website also provided aggregated compliance rates across different driving environments (urban, rural, and school zones).

\section{Driving Report Overview}
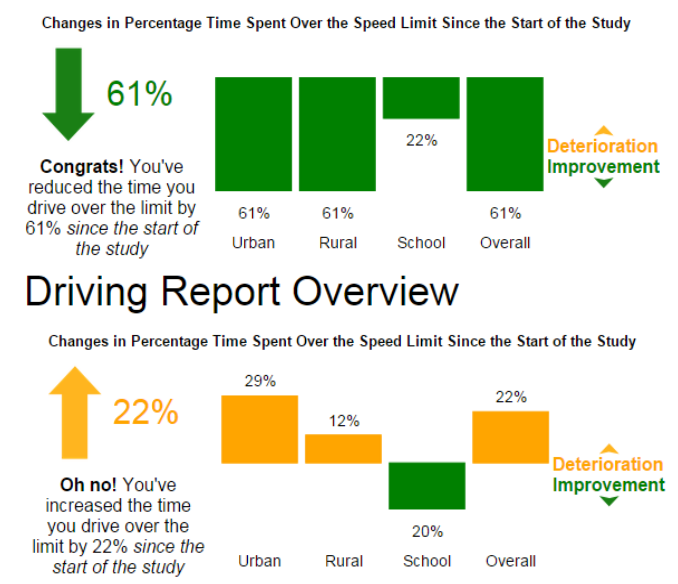

Weekly Summary of Time Spent Over the Speed Limit

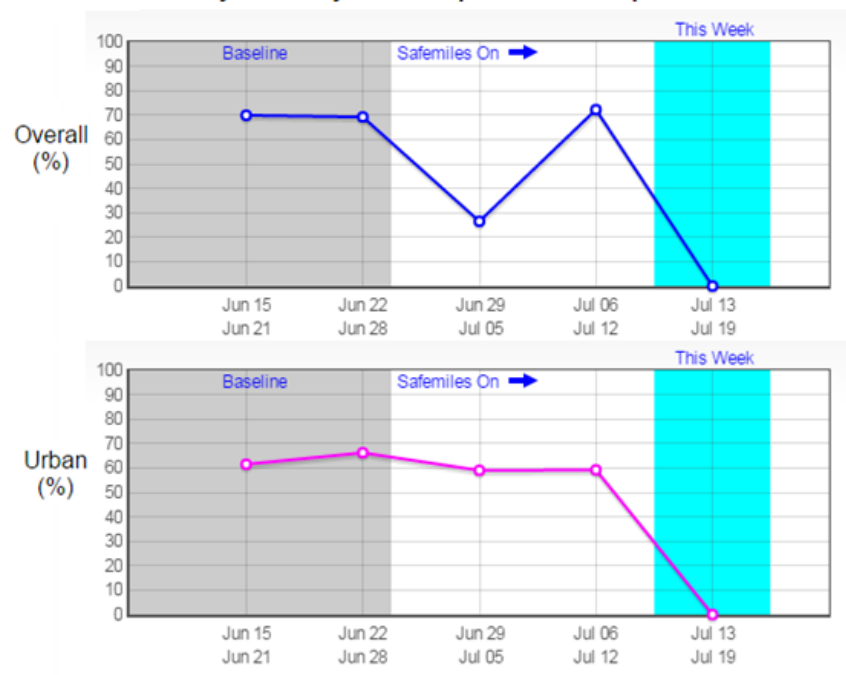

Fig. 3. Examples of visualizations shown on the post-drive feedback website. Top: overall changes in time spent over the speed limit, depicting (i) an overall increase in speed limit compliance, with improvements in all environments, and (ii) an overall reduction in speed limit compliance, but with improvements in school zone only. Bottom: trends of percent time spent over speed limit on a weekly basis in the urban environment. In gray was the last two weeks of the baseline period. A text label "Safemiles On" showed participants since when the Safemiles system had been turned on. The current week was highlighted in blue. Similar graphs were presented for rural and school zones.

\section{F. Procedures}

Participants who met the study criteria were required to complete an online information tutorial to ensure that they understood the information about their participation. Based on the condition they were assigned to, the participants were introduced to the "SafeMiles System" only or the "SafeMiles System" plus the "SafeMiles Website" in this tutorial. Participants were made aware that vehicle data was being collected throughout the study, while feedback/incentives were only available during the intervention period. Furthermore, participants were given the following instruction regarding the naturalistic characteristic of this study: "This is a naturalistic driving behaviour research, which means we are interested in how people drive in their natural environment. We ask that you drive the vehicle in your usual manner and for your normal purposes during the study. Your safety should always remain a priority. There is no need to interact with the SafeMiles system manually while you are driving." 
Informed consent was obtained during vehicle instrumentation. On-line questionnaires were administered at multiple points in the study. A screening questionnaire collected self-reported data on demographics, vehicle characteristics, and vehicle use. Study participants were surveyed about their driving behaviours and relevant personality factors during the baseline period and were probed about their experience and opinions regarding the intervention they experienced after the intervention period. At the end of data collection, in-vehicle equipment was removed from the vehicles and participants were debriefed.

\section{RESULTS}

Participants together travelled a total of $298,370 \mathrm{~km}$ over the entire study. While data collection took place in both urban (City of Winnipeg) and rural (surrounding regions of Winnipeg) environments, this paper reports on data collected within the urban environment only-including the perimeter highway that forms a beltway around the city of Winnipegdue to the relatively low travel rate in the rural environment $(61,833 \mathrm{~km}$ in total, or roughly $20 \%$ of data collected). Similarly, data collected in $30(1603 \mathrm{~km}), 40(0 \mathrm{~km})$, and 90 (4204 km) km/h speed limit zones within the urban environment were excluded from the analysis due to the relatively few numbers of observations. Furthermore, the analysis excluded data collected during the first two weeks of the baseline to ensure participants had sufficient time to adapt to the device installed in their vehicle.

\section{A. Dependent Measures and Statistical Models}

Speed limit compliance rate over a given period of time was defined as the ratio of the compliant time (GPS based speed $\leq$ posted speed limit $+5 \mathrm{~km} / \mathrm{h}$ ) over the total time spent driving in a particular speed limit zone. In total, speed limit compliance rates were computed for five speed limit zones (50, 60, 70, 80, and $100 \mathrm{~km} / \mathrm{h}$ in the urban environment), over four experimental phases: baseline (weeks $3 \& 4$ ), intervention-1 (weeks 5 - 9), intervention-2 (weeks 10 - 14), and postintervention (weeks $15 \& 16$ ). The ten weeks of intervention data were analyzed in two phases (intervention-1 and intervention-2) to help differentiate between initial response to and later effects of interventions. These computations did not include vehicle idle time or parked time as part of the compliant time or the total time spent driving to obtain a more accurate estimate of compliance rates. However, the present analysis did not account for slowdowns due to heavier traffic or traffic infrastructure (e.g., slowing down prior to reaching a stop sign).

Data collected in the $100 \mathrm{~km} / \mathrm{h}$ speed limit zone essentially comprised of driving on the perimeter highway surrounding the City of Winnipeg. As highway driving may be considerably different from non-highway driving (e.g., no stops from traffic lights or stop signs), the $100 \mathrm{~km} / \mathrm{h}$ speed limit zone was analyzed separately from the other speed limit zones.

For all data but the $100 \mathrm{~km} / \mathrm{h}$ speed zone, i.e., non-highway urban environment, a linear mixed-effects model [22] was built in SAS to analyze speed limit compliance rates. Feedback type (real-time, real-time \& financial incentives, and real-time \& post-drive), experimental phase (baseline, intervention-1, intervention-2, and post-intervention), and speed limit zone (50,
60, 70, and $80 \mathrm{~km} / \mathrm{h}$ ) were treated as fixed factors, and participant as a random factor. Total driving time in a given speed limit zone and experimental phase was included as a covariate to control for exposure to different speed limits in different experimental phases. Non-significant interaction terms $(\alpha>$.05) were dropped from the final model through backward selection, except for the interaction between experimental phase and feedback type, which was necessary for our planned contrasts.

For the highway data (i.e., $100 \mathrm{~km} / \mathrm{h}$ speed limit zone), the normality assumption of residuals was not met for the linear mixed-effects model, therefore, a mixed-effects beta regression with the logit link function was fitted instead in SAS. Beta regression is particularly well suited to non-binomial proportion variables, which take values in the interval $[0,1]$. As beta distribution is defined on the open unit interval $(0,1)$ only, 0.005 was subtracted from values at the upper bound of 1 (when a participant was compliant during an entire phase) [21]. Once again, feedback type and experimental phase were treated as fixed factors, participant as a random factor, and total driving time in a given experimental phase as a covariate.

For both models, a priori planned contrasts were used to examine changes in compliance rates within each feedback type:

- baseline to intervention-1

- baseline to intervention-2

- intervention-1 to intervention-2

- intervention-2 to post-intervention

- baseline to post-intervention.

Planned contrasts were also used to control for potential baseline differences across participants when comparing the effectiveness of feedback types: i.e., the magnitude of changes in compliance rates from baseline to intervention-1, to intervention-2, and to post-intervention, respectively, were compared across feedback types.

For the beta regression, the effect ratios (ERs) obtained through planned contrasts are similar to odds ratios given that a logit link function was used. However, because compliance rate was modeled rather than compliance probability, ERs obtained in this beta regression model are not equivalent to odds ratios. Rather, the ER comparing two conditions (e.g., A vs. B) can be written as follows with rate replacing probability in the odds ratio equation:

$$
E R=\frac{\frac{\text { Compliance rate }}{1-\text { Compliance rate }} \mid \text { Condition } A}{\frac{\text { Compliance rate }}{1-\text { Compliance rate }} \mid \text { Condition } B}
$$

\section{B. Speed Limit Compliance in Non-Highway, Urban Speed Limit Zones}

Speed limit compliance rates during baseline were around $75 \%$ and were consistent across the three feedback types, $F(2$, $55)=0.48, p=.62$, see Fig. 4. Speed limit zone was a significant factor, $F(3,165)=14.3, p<.0001$. Compliance rates in the 60 $\mathrm{km} / \mathrm{h}$ speed limit zone were significantly higher by $2.51 \%$ [95\% Confidence Interval: $1.25,3.78$ ] compared to the $50 \mathrm{~km} / \mathrm{h}$ zone and by $3.82 \%[2.54,5.11]$ compared to the $80 \mathrm{~km} / \mathrm{h}$ zone. The $70 \mathrm{~km} / \mathrm{h}$ zone also produced $2.64 \%$ [1.41, 3.86] higher compliance rates compared to the $80 \mathrm{~km} / \mathrm{h}$ zone. The interaction 
effect between experimental phase and feedback type was approaching but not significant, $F(6,159)=1.86, p=.09$, whereas the main effect of experimental phase was significant, $F(3,159)=5.19, p=.002$.

A priori planned contrasts showed that for those who received real-time feedback only, speed limit compliance rates did not improve significantly from baseline to intervention-1, but increased by $2.68 \%[0.51,4.86]$ from baseline to intervention-2. There were no significant changes from intervention-1 to intervention-2, from intervention- 2 to postintervention, and from baseline to post-intervention.

Within the financial incentives group, speed limit compliance rates increased by $4.15 \%$ [1.97, 6.32] from baseline to intervention-1, and by $2.69 \%$ [0.52, 4.85] from baseline to intervention-2. While there was no significant change from intervention- 1 to intervention-2, there was a $2.48 \%$ [0.30, 4.66] drop from intervention-2 to post-intervention. Once again, no significant changes were observed in compliance rates between baseline and post-intervention.

For the post-drive feedback group, no significant changes in compliance rates were observed in any of the planned contrasts. Supporting the above results, we also found that the difference between intervention-1 and the baseline was 3.24\% [0.28, 6.20] smaller in post-drive feedback group, compared to the financial incentives group.

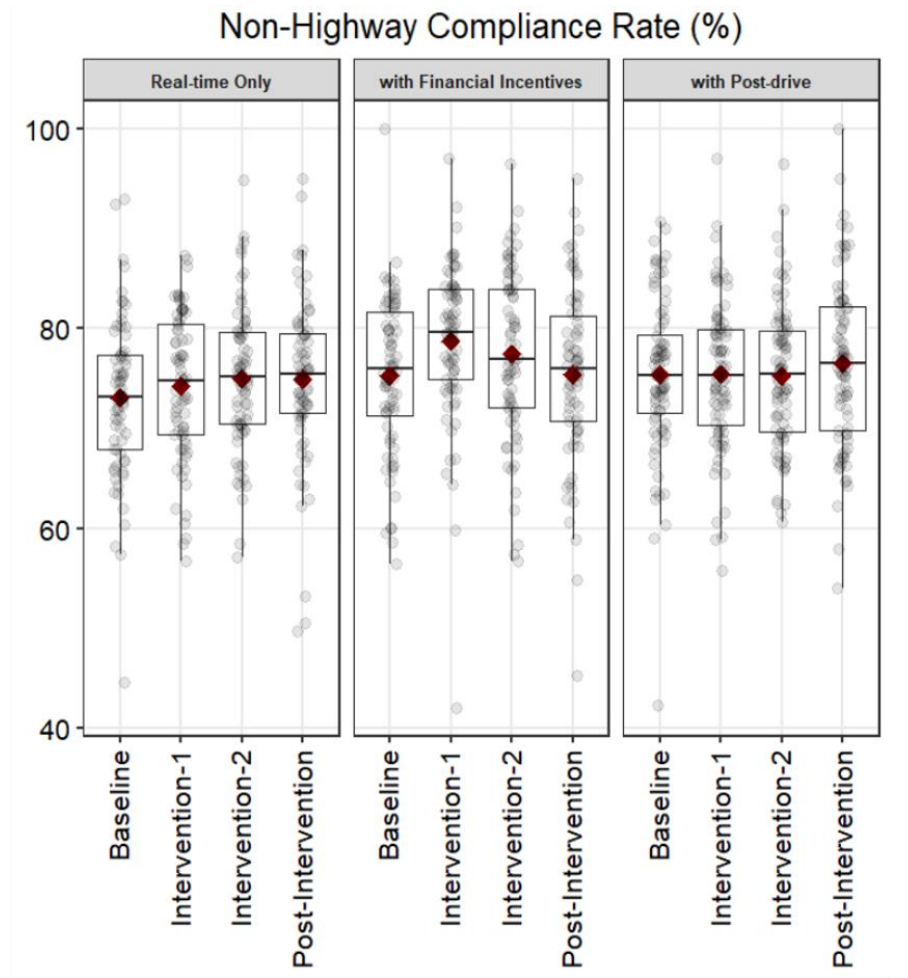

\begin{tabular}{|l|r|r|r|r|r|r|r|r|r|r|r|r|}
\hline Mean & 73.1 & 74.3 & 75.0 & 75.0 & 75.3 & 78.7 & 77.4 & 75.4 & 75.4 & 75.4 & 75.3 & 76.5 \\
\hline SD & 8.0 & 7.3 & 7.2 & 8.3 & 7.9 & 7.9 & 8.5 & 8.9 & 7.9 & 7.5 & 7.3 & 8.6 \\
\hline
\end{tabular}

Fig. 4. Urban, non-highway zones: Speed limit compliance rates across experimental phases by feedback type. Boxplots represent the range, 1st quartile, median, and 3rd quartile. Gray circles are observations, per person, averaged over each speed limit zone. Red diamonds represent the means. The mean (M) and standard deviation (SD) values are listed below each condition.

\section{Speed Limit Compliance in Highway Environment}

Speed limit compliance rates during baseline were around $60 \%$ for all three feedback types, with large variability across participants, $F(2,43)=0.05, p=.95$, see Fig. 5 . There was a significant interaction effect between experimental phase and feedback type, $F(6,109)=3.21, p=.006$, in addition to significant main effects of experimental phase, $F(3,109)=9.84$, $p<.0001$, and feedback type, $F(2,39)=4.10, p=.02$.

A priori planned contrasts showed an improvement in compliance rates from baseline to intervention-1 in both the real-time only feedback and the financial incentives groups: ER: 2.35 [1.17, 4.69] and 7.20 [2.92, 17.8], respectively. Significant improvements were also found from baseline to intervention-2 for these two groups, ER: 2.83 [1.42, 5.66] and $7.14[2.89,17.6]$, respectively.

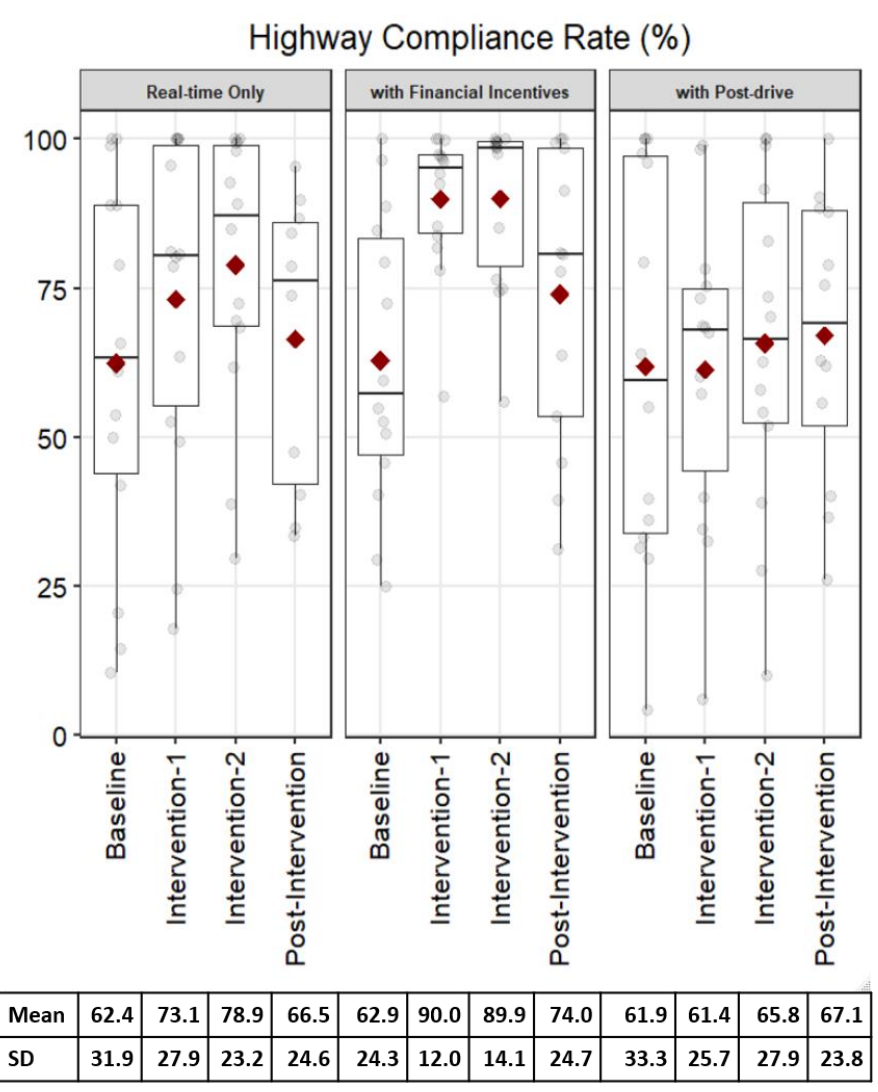

Fig. 5. Highway zones: Speed limit compliance rates across experimental phases by feedback type. Boxplots represent the range, 1st quartile, median, and 3rd quartile. Gray circles are observations, per person, averaged over each speed limit zone. Red diamonds represent the means. The mean (M) and standard deviation (SD) values are listed below each condition.

Controlling for baseline differences, the group receiving financial feedback showed a larger change in compliance between intervention-1 and baseline compared to the group receiving only real-time feedback, ER: 3.07 [1.02, 9.22], and compared to the group receiving post-drive feedback, ER: 6.76 $[2.31,19.8]$. The difference between intervention-2 and baseline was also larger for the financial incentives group compared to the post-drive feedback group, ER: 5.53 [1.88, 16.3]. However, these improvements did not seem to sustain after feedback/incentives were removed. Compliance rates deteriorated from intervention-2 to post-intervention 
significantly in the financial incentives group and in the realtime feedback only group, ER: $0.21[0.08,0.53]$ and 0.44 [0.204 0.97], respectively.

No significant changes in speed limit compliances were found across the four phases in the post-drive feedback group. Overall, there were no significant differences in compliance rates between baseline and post-intervention in any of the feedback groups.

\section{Post-drive Website Access Frequency and Speed Limit Compliance}

Contrary to our expectations, post-drive feedback was not effective in raising speed limit compliance. However, there were differences observed in how often participants assigned to the post-drive feedback group accessed the feedback website, despite the financial incentives offered (up to \$100) for accessing it regularly. A number of participants did not log in at all or, on average, logged in less than once per week. Over the 10-week intervention period, participants accessed the website between 0 and 38 times $(M=9.95, S D=9.52)$. Given that those who did not access or rarely accessed the website would not have been fully exposed to the potential benefits of accumulated post-drive feedback, we attempted to explore the relation between speed limit compliance and login frequency of feedback website through correlation analysis.

TABLE I

CORRELATIONS BETWEEN WEBSITE LOGIN FREQUENCY DURING THE INTERVENTION PERIOD AND SPEED LIMIT COMPLIANCE CHANGES BETWEEN PHASES

\begin{tabular}{|l|c|c|}
\hline \multirow{2}{*}{ Compliance Change from } & \multicolumn{2}{|c|}{ Spearman's $\rho$, p-value } \\
\cline { 2 - 3 } & $\begin{array}{c}\text { Non-highway } \\
\text { Data }\end{array}$ & $\begin{array}{c}\text { Highway } \\
\text { Data }\end{array}$ \\
\hline Baseline to Intervention1 & $\rho=0.11, p=.66$ & $\rho=0.57, p=.19$ \\
\hline Baseline to Intervention2 & $\rho=0.02, p=.94$ & $\rho=0.63, p=.23$ \\
\hline Baseline to Post-intervention & $\rho=-0.11, p=.65$ & $\rho=0.34, p=.29$ \\
\hline Intervention2 to Post-intervention & $\rho=-0.06, p=.80$ & $\rho=-0.04, p=.90$ \\
\hline
\end{tabular}

Table 1 reports Spearman correlations between the frequency of website access during the intervention period and the speed limit compliance changes observed across different phases. While none of the correlations were significant, likely in part due to the small sample size, some values may be noted for substantial correlation coefficients. In particular, higher frequency of website access was associated with larger values of compliance improvements for highway driving from baseline to intervention-1 $(\rho=0.57)$ and to intervention-2 $(\rho=0.63)$.

\section{E. Subjective Responses on Intervention Experienced}

After the ten-week intervention phase, participants completed an online questionnaire that probed their experiences with the different components of the intervention they experienced. Majority of the participants $(79.3 \%$ or $n=46$ out of 58 total) held a positive opinion about in-vehicle feedback (including trip summary for the post-drive group) they experienced, and $65.5 \%$ of participants $(n=38)$ agreed that invehicle feedback enhanced their driving safety. The same participants $(79.3 \%$ or $n=46)$ reported increased awareness of posted speed limits after the intervention period as a result of feedback. Many participants $(75.9 \%$ or $n=44)$ stated that they would use this in-vehicle feedback system if it comes with the vehicle at no additional cost, and without any financial incentives. When probed about real-time feedback in particular, 44 participants $(75.9 \%)$ thought that real-time feedback helped increase their compliance.

One factor that may limit how useful participants find a feedback system is their perceived ability to drive safely without it. All participants felt neutral about or agreed with (62.1\% or $n=36$ strongly agreed with) the statement "I am confident in my ability to drive the car safely without the SafeMiles System." In retrospect, referring to the ability to "drive safely" may be too general for the purpose of the feedback examined. An item on the driver's ability to comply with speed limits without the feedback system may have elicited more relevant opinions. Perceived accuracy of the system might also play a role in how useful participants find a feedback system: $10.3 \%$ of participants $(n=6)$ were under the impression that the in-vehicle feedback system only "sometimes" identified the correct speed limit. It is difficult to assess whether the system was actually incorrect about the speed limit at times, or this perception reflected a participant's inaccurate mental model about speed limits. The speed limit maps built into the system were based on government-issued reports, and comprehensively tested by the contracting engineers who drove through all roads on the map.

Financial gains appeared to be the most important motivator for speed limit compliance. Not surprisingly, participants who received financial incentives cited the following reason for reducing their speed during the intervention period most frequently (more than one choice was allowed): "Because I wanted to receive financial bonus provided in the study" (73.7\% of the financial incentives group). The second most cited reason was "Because I did not want to get a fine for speeding" $(57.9 \%)$. For the other two experimental groups, the most frequently selected response was fine avoidance $(63.2 \%$ of the real-time feedback only group, and $75.0 \%$ of the postdrive feedback group) and safety: "Because I thought it was safer to drive at or under the posted speed limit" was the second most common response selected by the real-time only $(57.9 \%)$ and post-drive feedback (40.0\%) groups.

Participants were also probed for reasons to not reduce their speed when real-time feedback indicated that they were over the speed limit. The most frequently cited reason across all feedback groups was "Because I felt my speed was still safe" (62.1\% of participants), followed by "Because I was in a rush to get somewhere" $(46.6 \%)$.

TABLE II

USEFULNESS, SATISFYING, AND TRUST SCALES ACROSS PARTICIPANTS AND FEEDBACK CONDITIONS: DESCRIPTIVE STATISTICS

\begin{tabular}{|l|c|c|c|c|}
\hline \multirow{2}{*}{ Scale } & \multicolumn{4}{|c|}{ Mean, Standard Deviation } \\
\cline { 2 - 5 } & $\begin{array}{c}\text { Overall } \\
(\mathrm{n}=58)\end{array}$ & $\begin{array}{c}\text { Real-time } \\
(\mathrm{n}=19)\end{array}$ & $\begin{array}{c}\text { Real-time \& } \\
\text { financial } \\
(\mathrm{n}=19)\end{array}$ & $\begin{array}{c}\text { Real-time \& } \\
\text { post-drive } \\
(\mathrm{n}=20)\end{array}$ \\
\hline Usefulness & $0.93,0.82$ & $1.17,0.68$ & $0.68,0.92$ & $0.92,0.80$ \\
\hline Satisfaction & $0.32,1.00$ & $0.70,0.97$ & $-0.14,1.10$ & $0.41,0.80$ \\
\hline Trust & $4.07,0.58$ & $4.06,0.58$ & $4.00,0.54$ & $4.15,0.62$ \\
\hline
\end{tabular}

Questionnaire items from existing scales were included to gain further insights on participants' experience with the "SafeMiles System", in particular system acceptance [23], which had two subscales: 'usefulness' and 'satisfaction' (each an average of four Likert scale items with range -2 to 2), and 
trust [24] (average of six Likert scale items with range 1 to 5). Higher values of the scales correspond to more positive attitudes toward a system. Overall, the participants had a generally positive response to the system (see Table II). Interestingly, participants receiving financial incentives rated the system the lowest in all three scales. Linear models were built to analyze the three scales as a function of feedback group. Feedback group had a significant effect on the satisfying scale, see Fig. $6, \mathrm{~F}(2,55)=3.77, \mathrm{p}=.03$, where participants in the real-time feedback group rated the feedback system higher by $0.85[0.09,1.59]$, compared to those receiving both real-time and financial incentives. Feedback group was not a significant factor for the usefulness scale, $\mathrm{F}(2,55)=1.72, \mathrm{p}=.19$, or the trust scale, $\mathrm{F}(2,55)=0.33, \mathrm{p}=.72$.

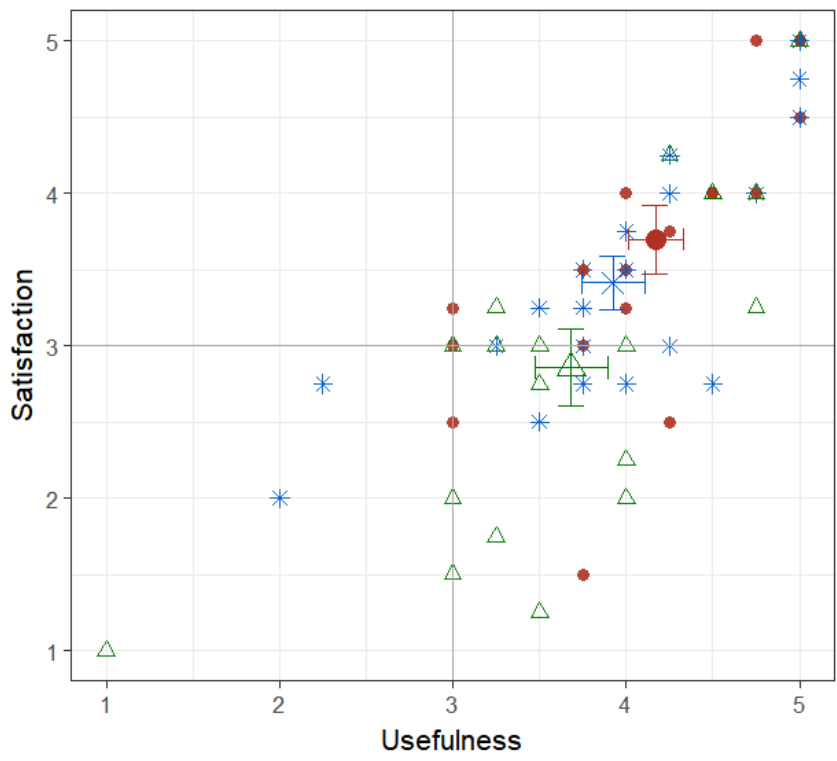

Real-time $\triangle$ Real-time \& financial incentives $*$ Real-time and Post-drive

Fig. 6. Means and standard errors of satisfaction and usefulness scales for the three feedback groups, plotted over individual scores.

\section{DISCUSSION}

In general, lower speed limit compliance rates, with larger variability among participants, were observed in highway driving compared to driving within the non-highway urban environment. Within each environment, speed limit compliance during baseline was fairly consistent among participants assigned to three different feedback conditions suggesting that the random participant assignment to the three conditions worked as intended.

\section{A. Impact of Financial Incentives}

Consistent with previous literature, our findings showed that real-time feedback as in [6],[7], [25], especially when combined with financial incentives as in [15], [16], [26], was effective in raising speed limit compliance rates. The advantage of real-time feedback and financial incentives was especially apparent in the highway environment, where the speed limit is high and there is less traffic infrastructure (e.g., traffic lights) to slow drivers down. However, contrary to [15], [16], which found sustained effects of improved speed limit compliance even after the feedback and financial incentives were removed, we did not observe significant differences in speed limit compliance between baseline and post-intervention periods for neither the real-time feedback only condition nor the real-time feedback + financial incentives condition. In the studies where improvements were sustained after intervention, participants were able to track both their performance and financial gain/loss through a web-based post-drive feedback system [15], [16], which made it impossible to asssess whether it was the addition of financial incentives, the addition of post-drive feedback, or the addition of both that drove the results.

\section{B. Efficacy of Post-Drive Feedback}

In our study, we carefully separated the financial incentives from post-drive feedback to assess the lone benefits of providing post-drive feedback on speed limit compliance. Surprisingly, our post-drive feedback did not lead to any speed reduction benefits. In a descriptive sense, we observed that those in the post-drive feedback group who were highly compliant during the baseline noticeably declined in compliance after the baseline period, while the less compliant participants showed to some extent positive changes in compliance during intervention and post-intervention. While our sample size is too small for statistical analysis that could properly account for a split in low/high compliance rates during baseline, future work may seek to understand whether there is also potential for the highly compliant participants receiving post-drive feedback to experience a 'boomerang' effect - that the aggregated feedback about how well they were doing might have lowered their efforts in maintaining their performance.

Another potential explanation was that many of the participants did not make use of the post-drive information available to them - nearly half the participants in this condition never accessed the website $(n=3)$ or accessed it 9 times or less $(\mathrm{n}=11)$, during the 10 week period. Although not statistically significant, positive changes in speed limit compliance from before to after feedback appeared to be associated with higher frequency of website access (a correlation coefficient of 0.63). This correlaton provides some, albeit limited, support to the efficacy of post-drive, information-based feedback that is not tied to financial incentives. Beyond increasing sample size, further research of post-drive feeedback can control for baseline speeding tendency in experimental design, and investigate the type of access to post-drive feedback (e.g., reinforced or selfinitiated). It would also be worthy to investigate driver characteristics that may explain the level of engagement participants have with post-drive feedback. For example, in an earlier study [15] that also took place in Winnipeg, participants who exhibited a lower speed limit compliance rate during the baseline were found to have significant improvements in the intervention period, during which they had access to a website for tracking both their driving performance and accumulated financial rewards. It may be that drivers are aware of their own speeding tendencies, and would calibrate their use of the postdrive feedback system accordingly. While Warner and Åberg [9] suggested that economic incentives tied to the use of a feedback system can result in a more sustained improvement with the system, the anticipated usefulness of a post-drive feedback system may also play a role in system use.

We also note that in our analysis, the level of engagement with post-drive feedback (website access) did not correlate with 
income or education level of the participants, but this lack of relationship may also be a result of the limited sample size. As noted earlier, our participants were limited to the age range of 25 to 40 years to control for potential differences related to driver age. Future studies should investigate the efficacy of different feedback types and incentives for different age groups.

\section{Feedback Acceptance}

Participants generally rated the SafeMiles system (i.e., invehicle feedback) highly on the satisfying, usefulness, and trust scales. Interestingly, the addition of financial incentives significantly lowered how satisfying participants found the realtime feedback to be, given the otherwise identical feedback experienced. Those receiving financial incentives also reported the lowest intention among the three feedback groups to use the feedback system without any further incentives. While initially enhancing speed limit compliance, financial incentives may produce unintended psychological consequences that could undermine potential benefits real-time feedback may have in the long run. Psychological research has long cautioned the use of extrinsic rewards on undermining free-choice intrinsic motivation as people may, for example, attribute their behaviour to the external reward rather than to their interest in the activity, i.e., the overjustification effect [26].

Questionnaire responses also revealed reasons for and against speeding. Consistent with literature on speeding behaviours [27], perceived risk ("Because I felt my speed was still safe") and benefits ("Because I was in a rush to get somewhere") were both top stated facilitators of speeding in our study. These findings suggest that effective feedback systems should inform participants not only about their speed choices, but also the risks associated with their speed choices.

\section{Limitations}

Data collection took place between June and September in one year to control for seasonal effects. A longer intervention period may be particularly useful for investigating post-drive feedback, where richer data accumulated over time and over different seasons may be more meaningful to the participants. A longer post-intervention period would also be valuable for investigating the long-term impact of feedback in general. Further, our sample of participants may not be representative of the general driving population as the study was conducted in one city in Canada. Our sample may not even be representative of the City of Winnipeg. Given that recruitment mostly relied on online advertisements and electronic mailing lists of local communities, participants who volunteered for this study may have been more familiar with Internet technology, which was also one of the two platforms of our post-drive feedback. The financial incentives involved might have also attracted participants who are more motivated by monetary rewards. In reality, C\$300 over a few months may be unlikely via incentive programs offered by insurance companies or the government. It may be that the significant increases in speed limit compliance observed in the group receiving financial incentives during the first half of intervention were heavily influenced by this amount - a more modest financial incentive might not necessarily inspire such change. However, it is important to note that even with such a high incentive, improvements in speed limit compliance did not sustain over time and began to drop during the second half of the intervention period.

Driving behaviour might have also been influenced by the presence of experimental equipment and the knowledge about the focus of the study being on speed limit compliance. We also note that our analysis of feedback effectiveness utilized only the measure of speed limit compliance. Other driving performance measures, such as average speed reductions, may provide additional insights regarding the magnitude of behavioural changes. Our study was designed to examine the effectiveness of feedback on speed limit compliance, and thus emphasized compliance rates to our participants, rather than the specific speed choices they made. In the future, a naturalistic study of larger scope may consider other ways to collect relevant data (e.g., front-facing and in-vehicle cameras, traffic and weather data) to support the analysis of average speeds that can account for the surrounding traffic situations.

\section{CONCLUSIONS}

This paper presents findings from a naturalistic driving study that compared the efficacy of financial incentives and postdrive feedback in enhancing speed limit compliance, when presented in addition to real-time feedback.

The main findings are as follows:

1) Speed limit compliance rates were lower, with larger variability among participants, in highway driving compared to driving within the non-highway urban environment.

2) Both real-time feedback alone and when it was combined with financial incentives were effective in increasing speed limit compliance. While the addition of financial incentives appeared to have the largest positive impact on speeding reduction initially, the effects did not sustain when feedback and incentives were removed. Technology acceptance ratings revealed that financial incentives lowered the level of satisfaction participants experienced with real-time feedback, cautioning for the use of financial rewards or penalties in intervention strategies. More research is necessary on how external rewards may be implemented without undermining the long term intrinsic motivation for safer driving behaviours.

3) Post-drive feedback did not show any positive influence. However, its usefulness may be limited by the fact that most of our participants did not access the website very much, despite the financial incentives to do so. Mechanisms to increase post-drive feedback adoption should be investigated alongside the usefulness of such information. For example, future work may investigate the use of gamification to tap into intrinsic motivation, deliver post-drive feedback via a more convenient platform, or consider an adaptive feedback system that tailors to the driver's characteristics, preferences, and progress over time (e.g., setting personal goals, providing incentives catered to a person's interest).

While our findings are specific to mitigating speeding behaviours, they may also inform the design of feedback systems for other forms of unsafe driving behaviours, such as 
distraction engagement. Post-drive feedback regarding driver performance and behavioural metrics have already been implemented by many insurance companies, where financial incentives in the form of insurance premium reduction are offered. However, further research is necessary to understand what information should be presented and how best to present it to achieve lasting effects of interventions.

\section{ACKNOWLEDGMENT}

Many thanks to James Foley, Kazu Ebe, and Chuck Gulash from Toyota CSRC for their feedback on this paper as well as providing directions for our research in general. We would also like to acknowledge the assistance of Ryan Kealey, Jeffrey Alam, Tony Ye, and Isaac Chung in conducting this study.

\section{REFERENCES}

[1] S. Klauer, J. Sudweeks, J. S. Hickman, and V. L. Neale, "How risky is it? An Assessment of the Relative Risk of En gaging in Potentially Unsafe Driving Behaviors," Virginia Tech. Transp. Inst., Blacksburg, VA, 2006.

[2] D. M. Neyens and L. N. Boyle, "The effect of distractions on the crash types of teenage drivers," Accident Analysis \& Prevention, vol. 39, no. 1, pp. 206-212, 2007.

[3] National Center for Statistics and Analysis, "2016 Fatal Motor Vehicle Crashes: Overview," National Highway Traffic Safety Administration, Washington, DC, Report DOT HS 812 456, Aug. 2017.

[4] R. Elvik, T. Vaa, A. Hoye, and M. Sorensen, The handbook of road safety measures. Emerald Group Publishing, 2009.

[5] A. H. Goodwin, L. J. Thomas, W. L. Hall, and M. E. Tucker, "Countermeasures that work: A highway safety countermeasure guide for state highway safety offices," 2010. [Online]. Available: https://trid.trb.org/view.aspx?id=915602.

[6] E. Adell, A. Várhelyi, and M. Hjälmdahl, "Auditory and haptic systems for in-car speed management-A comparative real life study," Transportation Research Part F: Traffic Psychology and Behaviour, vol. 11 , no. 6, pp. 445-458, 2008.

[7] K. Brookhuis and D. de Waard, "Limiting speed, towards an intelligent speed adapter (ISA)," Transportation Research Part F Traffic Psychology and Behaviour, vol. 2, no. 2, pp. 81-90, 1999.

[8] M. A. Regan and K. L. Young, "Effects on driving performance of invehicle intelligent transport systems: Final results of the Australian TAC SafeCar project.," in Proceedings of the 2005 Australasian Road Safety Research, Policing, and Education Conference, Wellington, New Zealand, 2005, pp. 157-162.

[9] H. W. Warner and L. Åberg, "The long-term effects of an ISA speedwarning device on drivers' speeding behaviour," Transportation Research Part F: Traffic Psychology and Behaviour, vol. 11, no. 2, pp. 96-107, 2008.

[10] L. N. Boyle and F. Mannering, "Impact of traveler advisory systems on driving speed: some new evidence," Transportation Research Part C: Emerging Technologies, vol. 12, no. 1, pp. 57-72, Feb. 2004.

[11] F. Lai and O. Carsten, "What benefit does Intelligent Speed Adaptation deliver: A close examination of its effect on vehicle speeds," Accident Analysis \& Prevention, vol. 48, pp. 4-9, Sep. 2012, doi: 10.1016/j.aap.2010.01.002.

[12] N. W. Mullen, H. Maxwell, and M. Bedard, "Decreasing driver speeding with feedback and a token economy," Transportation research part F: traffic psychology and behaviour, vol. 28, pp. 77-85, 2015.

[13] I. J. Reagan, J. P. Bliss, R. V. Houten, and B. W. Hilton, "The effects of external motivation and real-time automated feedback on speeding behavior in a naturalistic setting," Human Factors, vol. 55, no. 1, pp. 218-230, 2012.

[14] C. Dijksterhuis, B. Lewis-Evans, B. Jelijs, D. de Waard, K. Brookhuis, and O. Tucha, "The impact of immediate or delayed feedback on driving behaviour in a simulated Pay-As-You-Drive system," Accident Analysis \& Prevention, vol. 75, no. Supplement C, pp. 93-104, Feb. 2015, doi: 10.1016/j.aap.2014.11.017.
[15] M. Merrikhpour, B. Donmez, and V. Battista, "A field operational trial evaluating a feedback-reward system on speeding and tailgating behaviors," Transportation Research F: Traffic Psychology and Behaviour, vol. 27, pp. 56-68, 2014.

[16] J. W. Bolderdijk, J. Knockaert, E. M. Steg, and E. T. Verhoef, "Effects of Pay-As-You-Drive vehicle insurance on young drivers' speed choice: Results of a Dutch field experiment," Accident Analysis \& Prevention, vol. 43, no. 3, pp. 1181-1186, 2011.

[17] T. Toledo and T. Lotan, "In-vehicle data recorder for evaluation of driving behavior and safety," Transportation Research Record, vol. 1953, pp. 112-119, 2006.

[18] I. van Schagen and F. Sagberg, "The potential benefits of naturalistic driving for road safety research: Theoretical and empirical considerations and challenges for the future," Procedia-social and behavioral sciences, vol. 48, pp. 692-701, 2012.

[19] "Census metropolitan area of Winnipeg, Manitoba," Statistics Canada, Ottawa, Ontario, Data products, 2016 Census. Statistics Canada Catalogue no. 98-404-X2016001, 2017. Accessed: Jul. 14, 2015. [Online].

[20] D. Shinar, E. Schechtman, and R. Compton, "Self-reports of safe driving behaviors in relationship to sex, age, education and income in the US adult driving population," Accident Analysis \& Prevention, vol. 33, no. 1, pp. 111-116, Jan. 2001.

[21] P. Schroeder, L. Kostyniuk, M. Mack, and S. Abt, "2011 national survey of speeding attitudes and behaviors.," United States. National Highway Traffic Safety Administration. Office of Behavioral Safety Research, 2013.

[22] J. Pinheiro and D. Bates, Mixed-effects models in S and S-PLUS Springer Science \& Business Media, 2006.

[23] J. D. Van Der Laan, A. Heino, and D. De Waard, "A simple procedure for the assessment of acceptance of advanced transport telematics," Transportation Research Part C: Emerging Technologies, vol. 5, no. 1, pp. 1-10, 1997.

[24] J.-Y. Jian, A. M. Bisantz, and C. G. Drury, "Foundations for an empirically determined scale of trust in automated systems," International Journal of Cognitive Ergonomics, vol. 4, no. 1, pp. 5371, 2000.

[25] L. Harms, B. Klarborg, H. Lahrmann, N. Agerholm, E. Jensen, and N. Tradisauskas, "Controlled study of ISA effects: comparing speed attitudes between young volunteers and external controls, and the effect of different ISA treatments on the speeding of volunteers," IET Intelligent Transport Systems, vol. 2, no. 2, pp. 154-160, 2008.

[26] U. Mazureck and J. Hattem, "Rewards for Safe Driving Behavior: Influence on Following Distance and Speed," Transportation Research Record: Journal of the Transportation Research Board, vol. 1980, pp. 31-38, Jan. 2006, doi: 10.3141/1980-07. 